\title{
ARTICLE Fully inkjet-printed microwave passive electronics
}

\author{
Garret McKerricher $^{1, *}$, Mohammad Vaseem ${ }^{1, *}$ and Atif Shamim ${ }^{1}$
}

Fully inkjet-printed three-dimensional (3D) objects with integrated metal provide exciting possibilities for on-demand fabrication of radio frequency electronics such as inductors, capacitors, and filters. To date, there have been several reports of printed radio frequency components metallized via the use of plating solutions, sputtering, and low-conductivity pastes. These metallization techniques require rather complex fabrication, and do not provide an easily integrated or versatile process. This work utilizes a novel silver ink cured with a low-cost infrared lamp at only $80^{\circ} \mathrm{C}$, and achieves a high conductivity of $1 \times 10^{7} \mathrm{~S} \mathrm{~m}{ }^{-1}$. By inkjet printing the infrared-cured silver together with a commercial 3D inkjet ultraviolet-cured acrylic dielectric, a multilayer process is demonstrated. By using a smoothing technique, both the conductive ink and dielectric provide surface roughness values of $<500 \mathrm{~nm}$. A radio frequency inductor and capacitor exhibit state-of-the-art quality factors of 8 and 20, respectively, and match well with electromagnetic simulations. These components are implemented in a lumped element radio frequency filter with an impressive insertion loss of $0.8 \mathrm{~dB}$ at $1 \mathrm{GHz}$, proving the utility of the process for sensitive radio frequency applications.

Keywords: capacitor; filter; inductor; inkjet printing; multi-jet; radio frequency; silver

Microsystems \& Nanoengineering (2017) 3, 16075; doi:10.1038/micronano.2016.75; Published online: 30 January 2017

\section{INTRODUCTION}

One of the major advancements in inkjet printing has been the use of ultraviolet (UV)-cured acrylic materials. These liquid inks immediately solidify on exposure to a low-cost UV lamp, and, like acrylic paints, they can be made with vibrant colors. This concept has been extended beyond graphic arts by using hundreds of inkjet nozzles to form fully printed 3D acrylic parts in minutes. Although multi-jet technology is still in its infancy, commercial multi-jet printers are now available ${ }^{1}$. A major advantage of the $3 \mathrm{D}$ multi-jet process over other 3D printing methods, such as stereolithography, selective laser sintering, or fused deposition modeling, is that multiple materials can be easily deposited together, just as an inkjet head routinely prints with cyan, magenta, yellow and black. Thus far, multi-jet printing has been restricted to the colorful UV-cured acrylics and wax/gel support materials. Fully printing objects with an integrated highconductivity metal provides exciting possibilities for additive and on-demand fabrication of radio frequency electronics such as inductors, capacitors, and filters.

To date, there have been several reports of printed objects incorporating metal, focused on RF applications, by using plating solutions, aerosol, sputtering, and low-conductivity pastes ${ }^{2-4}$. These metallization techniques require rather complex fabrication, and do not provide an easily integrated or versatile process. Previous reports have also put the spotlight on other issues with $3 \mathrm{D}$ printing techniques for electronics fabrication, such as micrometer surface roughness and low conductivity of the metal ${ }^{5}$. A recent report by $\mathrm{Wu}$ et al. shows a novel concept of $3 \mathrm{D}$ printing, together with syringe injection of a silver paste at $70{ }^{\circ} \mathrm{C}$, to create $3 \mathrm{D}$ inductors and capacitors; however, the conductive paste achieves a low conductivity of $2.8 \times 10^{5} \mathrm{~S} \mathrm{~m}^{-1}$, $\sim 200$ times less than that of bulk silver ${ }^{2}$. In our previous work, silver nanoparticles were utilized to metallize a 3D printed antenna, but the particles required selective laser sintering to avoid damaging the acrylic material and achieved a conductivity of only $1 \times 10^{6} \mathrm{~S} \mathrm{~m}^{-1}$ (Ref. 6). A major challenge of fully printing electronics is that the high temperature required for the metal is incompatible with the printed dielectric.

Most of the previous work on inkjet-printed Radio Frequency (RF) passives is focused on two-dimensional (2D) inkjet printing of metal on a standard substrate. Redinger et al. reported some of the first work on 2D printed capacitors and inductors in 2004, utilizing nanoparticles but achieving rather low quality factors (Q's) of $\sim 0.5$ (Ref. 7). Since then, there have been material and printing advancements enabling $2 \mathrm{D}$ printed inductors and capacitors with high-frequency Q's below 10 (Refs. 8-10). To the authors best knowledge, the combination of these fully printed components to design a GHz lumped element filter has never been demonstrated. Distributed 2D RF filters are generally simpler to print than lumped element filters. Previous distributed filter results have shown inadequate performance, with the insertion loss ranging from 3.6 to $10 \mathrm{~dB}$ (Refs. 11-15). One of the best reports has shown an insertion loss of $\sim 0.5 \mathrm{~dB}$ at its center frequency $(2 \mathrm{GHz})$, but it utilized inkjet printing along with electroless plating to increase the conductivity and thickness of the metal ${ }^{16}$.

This work provides a process beyond 2D inkjet printing of the conductor on a standard support substrate; the metal is truly integrated into the printed dielectric to build quality multilayer RF capacitors and inductors with crossover interconnects. A major advancement is the low processing temperature $\left(80^{\circ} \mathrm{C}\right)$ of the novel Silver-Organo-Complex (SOC) ink, which overcomes the major challenge of temperature processing compatibility between the printed conductor and dielectric. The SOC ink is cured with a

\footnotetext{
${ }^{1}$ King Abdullah University of Science and Technology (KAUST), IMPACT Lab, Computer, Electrical and Mathematical Sciences and Engineering (CEMSE) Division, Thuwal 239556900, Saudi Arabia.

Correspondence: Garret McKerricher (garret.mckerricher@kaust.edu.sa)

*These authors contributed equally to this work.

Received: 28 June 2016; revised: 7 September 2016; accepted: 8 September 2016
} 
low-cost IR lamp in only $5 \mathrm{~min}$, while providing state-of-the-art conductivity of $1 \times 10^{7} \mathrm{~S} \mathrm{~m}^{-1}$, which is necessary for conductivitysensitive RF filter applications.

\section{MATERIALS AND METHODS}

\section{Ultraviolet-cured dielectric ink}

Stratasys UV-cured ink is sold under the name Vero and comes in different colors and hardnesses. From the material safety data sheet of VeroWhite, it is composed mostly of acrylic monomers $(<30 \%)$, isobornyl acrylate $(<25 \%)$, and various other components, including acrylate oligomers and urethane acrylates ${ }^{17,18}$. Less than $1 \%$ titanium dioxide is used for the white color. The photoinitiator is diphenyl-2,4,6 trimethylbenzoyl oxide $(<2 \%)$, which produces a free radical on UV exposure, initiating the polymer reaction of the acrylic monomers and oligomers to form a hardened acrylic part. The acrylic monomers are liquid at room temperature but too viscous for jetting at $125 \mathrm{cp}$; see Figure $1 \mathrm{a}$.

Although the UV dielectric ink is tailored for the Stratasys Objet product line of 3D inkjet printers, it was more convenient to print with a Dimatix 2831 printer. The Dimatix printer allows control over nearly all print settings. By using a jetting temperature of $60^{\circ} \mathrm{C}$, the viscosity of the dielectric ink drops to $20 \mathrm{cp}$, as shown in Figure 1a, allowing for excellent jetting from the Dimatix 10-pL print head. The ink does not show any change in viscosity over the three different shear rate measurements shown in Figure 1a. The average mass of a single drop of the UV-cured ink jetted at $60^{\circ} \mathrm{C}$ and a $9-\mathrm{m} \mathrm{s}^{-1}$ velocity is $9.6 \mathrm{ng}$, measured by ejecting five million drops and weighing the total. Throughout this work, the UV-cured ink is printed with two layers at $30 \mu \mathrm{m}$, with drop spacing forming $11-\mu \mathrm{m}$-thick layers. For curing, it is exposed to $7500 \mathrm{~mJ} \mathrm{~cm}^{-2}$ of 365-nm-wavelength light after printing. After UV curing, thermogravimetric analysis was completed to understand the thermal limits of the material; see Figure 1b. The TGA shows that there is negligible mass loss up to $150{ }^{\circ} \mathrm{C}$; however, the material specification sheet reports the glass transition for the material $\sim 47-53^{\circ} \mathrm{C}$, and it was found experimentally that there is extreme warping of the material over $80^{\circ} \mathrm{C}$ (Ref. 19). Therefore, it is necessary to have a conductor that can be processed at temperatures under $80^{\circ} \mathrm{C}$.

To design radio frequency components, it is important to know the dielectric properties. From the parallel plate measurements (Figures 1c and d), the VeroWhite material has a dielectric constant of $\sim 3.0$ and a dissipation factor of $\sim 0.02$ up to $1 \mathrm{GHz}$. The dissipation factor of the material is rather high and can cause attenuation of the RF signal; however, FR-4 also has a dissipation factor of 0.02 and is the go-to material for low-cost RF applications ${ }^{20}$. Overall, this material is adequate for many RF designs but does have appreciable dielectric loss, and it has limitations in terms of temperature range $\left(<45^{\circ} \mathrm{C}\right)$ that should be respected.

\section{Infrared-cured silver-organo-complex ink}

The SOC ink utilized in this work has been developed in-house to overcome the issues with conventional metal nanoparticle ink. While silver nanoparticle ink has been widely investigated and is available commercially, it has a complex synthesis protocol, high cost, and high sintering temperature $\left(>150^{\circ} \mathrm{C}\right)$, and exhibits particle aggregation, nozzle clogging, a poor shelf life, and jetting instability. Through the use of smaller nanoparticles $(\sim 10 \mathrm{~nm})$ and a more robust ink formulation, commercial silver ink performance has improved considerably in recent years. However, in the long term, it is difficult to avoid particle aggregation and precipitation in a nanoparticle system. Organometallic inks are another approach to printing conductive patterns. They contain dissolved precursors of metallic elements bonded with organics (that is, silver acetate, silver oxalate, and copper hexanoate) ${ }^{21-23}$. In general, the organometallic bond is broken, and the organic molecule evaporates away, leaving a metal film behind. In the past, organometallics have been less successful than their
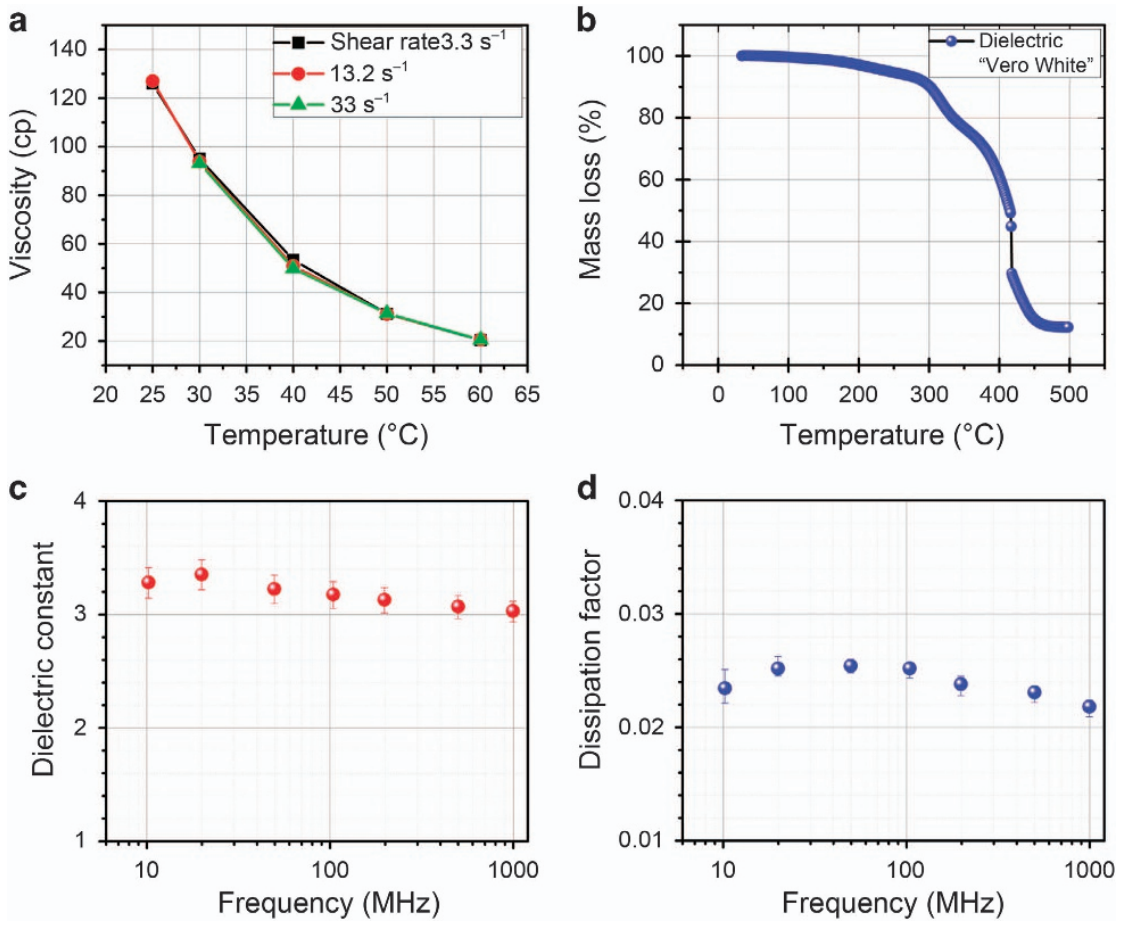

Figure 1 (a) Viscosity of VeroWhite dielectric ink. (b) TGA of VeroWhite material after UV curing. (c) Dielectric constant properties of VeroWhite material after UV curing. (d) Dissipation factor. Bars represent the maximum and minimum measurement values of five test samples with the parallel plate method Agilent E4991 and dielectric test fixture 16453A. 
Table 1 Fluid properties- $\mathrm{SOC}$ ink is measured at $25^{\circ} \mathrm{C}$ with a jetting velocity of $10 \mathrm{~m} \mathrm{~s}^{-1}$

\begin{tabular}{lcccccccc}
\hline Ink Type & Viscosity (cp) & Surface T. Dyne/cm & Density (g/cc) & Drop Mass (ng) & $(\mathrm{Oh})$ & $(\mathrm{Re})$ & $(\mathrm{We})$ & $(\mathrm{Ca})$ \\
\hline SOC Ink & 5.9 & 30.7 & 1.17 & 7.0 & 0.21 & 41.6 & 80.0 & 1.9 \\
VeroWhite Dielectric Ink & 20.0 & 30.2 & 1.1 & 9.6 & 0.75 & 10.4 & 62 & 6.0 \\
\hline
\end{tabular}

VeroWhite dielectric ink is measured at $60^{\circ} \mathrm{C}$ with a jetting velocity at $9 \mathrm{~m} \mathrm{~s}^{-1}$ velocity. Both inks utilize a Dimatix 10-pL DMC cartridge with a $21-\mu \mathrm{m}$-diameter nozzle. Ca, Capillary; Oh, Ohnesorge; Re, Reynolds; Surface T., Surface Tension; We, Weber.
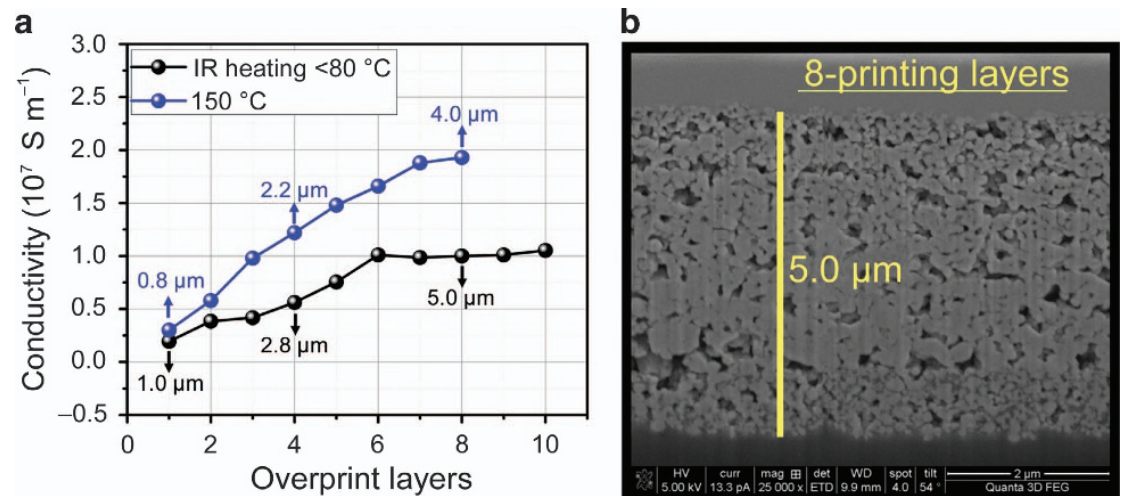

Figure 2 (a) Conductivity as a function of overprinting layers at different sintering conditions, that is, thermal heating at $150{ }^{\circ} \mathrm{C} / 30 \mathrm{~min}$ and IR heating at $<80^{\circ} \mathrm{C} / 5 \mathrm{~min}$. (b) SEM-focused ion beam cross-section of 8-layers of SOC ink with 5 min of IR $<80^{\circ} \mathrm{C}$ treatment after each overprint.

nanoparticle counterparts. One issue has been bubble formation, resulting in rough porous thin films as noted by Walker et al. ${ }^{24}$

An in-house SOC ink is utilized in this work, and it is capable of producing smooth and dense films; it is stable and transparent, with full details covered in the reference ${ }^{25}$. The SOC ink produces films with an impressive $1 \times 10^{7} \mathrm{~S} \mathrm{~m}^{-1}, 20 \%$ of the bulk conductivity at only $80^{\circ} \mathrm{C}$. Along with the high conductivity at low temperatures, the ink exhibits strong adhesion, long-term stability ( $>5$ months in a print head), and robust jetting performance. Briefly, the ink is based on a silver acetate complex with ethylamine, ethanolamine, water, methanol, and 2hydroxyethyl cellulose (HEC) (Mw 90 000), where the HEC acts as both viscosity modifier and adhesion promoter. The fluid parameters and operating points of both the SOC ink and the VeroWhite dielectric ink are given in Table 1. The Reynolds, Weber, Ohnesorge, and capillary number are provided since inks are often mapped by these parameters to fit in a specific jetting space. Although the SOC ink has a lower viscosity than the dielectric ink at 5.9 compared with $20 \mathrm{cp}$, both inks have good jetting stability and fit within the jetting space outlined by Derby et al. ${ }^{26}$

The conductivity of the SOC ink has been tested as a function of layer thickness, as shown in Figure 2a. The conductivity of the ink approaches $2 \times 10^{7} \mathrm{~S} \mathrm{~m}^{-1}$ at $150^{\circ} \mathrm{C}$ and $1 \times 10^{7} \mathrm{~S} \mathrm{~m}^{-1}$ at $80^{\circ} \mathrm{C}$ with increased overprints. Note that the $150^{\circ} \mathrm{C}$ heating was achieved on a glass substrate since the acrylic material would deform at temperatures above $80^{\circ} \mathrm{C}$. A low-cost 250 -watt IR lamp is used to cure the ink by putting the substrate under the light for five minutes after each printed layer. The maximum measured temperature of the substrate is $80^{\circ} \mathrm{C}$. This method was capable of achieving a high conductivity of $1 \times 10^{7} \mathrm{~S} \mathrm{~m}^{-1}$ by $\sim 6$ overprints. From the cross-section of Scanning Electron Microscopy (SEM) images (Supplementary Information Figure S1), there are clear voids in the printed film that are subsequently filled in by the overprints. More overprints result in a dense film with higher conductivity. Via 8 overprints and IR curing cycles, the film is fairly dense and $\sim 5-\mu$-thick, as seen in Figure $2 b$. Adhesion was a major concern for the ink, and it was found experimentally that the addition of $0.02 \mathrm{wt} \% 2$-HEC solved the issue, while increasing viscosity for superior jetting. Adhesion to glass, PET, and the $3 D$ printed materials was tested with scotch tape without any removal of the silver film. The ink is stable over the long term, as tested with a Dimatix 10-pL cartridge over five months with no observable reduction in the overall drop mass. Additionally, the printed films show no significant difference in conductivity after 10 months aging in environmental conditions (Supplementary Information Figure S2).

\section{Fabrication process}

A depiction of the complete inkjet process is shown in Figure 3. Two different printers were utilized, the Objet 3D multijet printer and Dimatix 2831 research printer, for convenience. The multijet printer allows quick processing of the UV material, while the Dimatix printer allows for more control over all of the print parameters and curing. It is easy to envision a single inkjet system or production line capable of performing all printing and curing. First, the UV cure material is printed with the Objet 3D printer, allowing for complex and thick shapes to be printed (i). After 3D printing, the substrate has several micrometers of surface roughness, as shown in Figure 4a. This roughness is substantial considering that the printed metal thickness itself is less than a micrometer per layer. Surface roughness is especially detrimental in RF components since it causes attenuation of the signal. The roughness issue is tackled by jetting an additional 'smoothing' layer of dielectric ink (Figure 4b) with the Dimatix 2831 inkjet printer and 10-pL head (ii). After deposition, the ink is allowed to spread before UV curing, and eventually, it smoothens the surface to $0.4 \mu \mathrm{m}$ of RMS roughness. SOC ink can then be printed on this smooth layer and cured with IR heating (iii). Next, in step (iv), the dielectric ink is printed with the Dimatix, and the dielectric ink covers the silver ink and is again cured with a UV lamp. Finally, in step (v), another layer of SOC ink can be printed, creating a multilayer process where the top and bottom layers are 


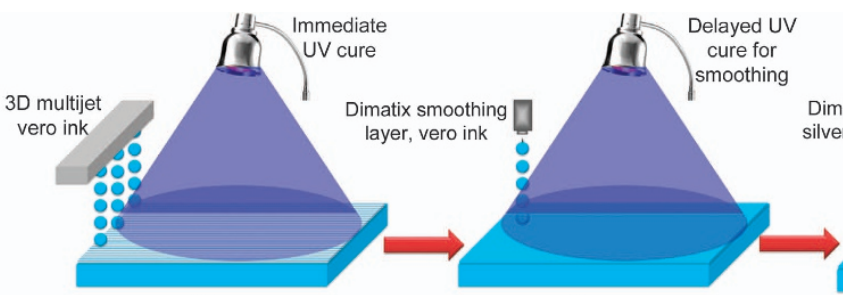

(i) (ii)

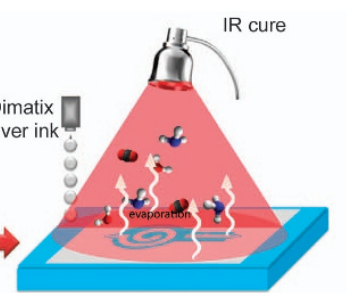

(iii)

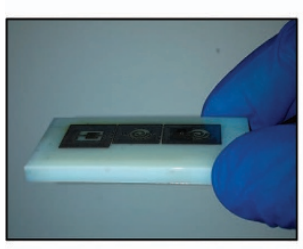

(vii)

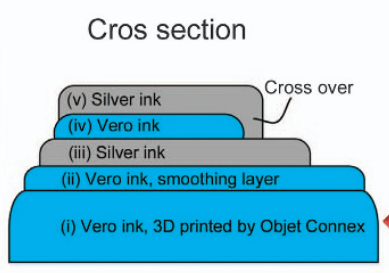

(vi)

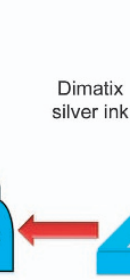

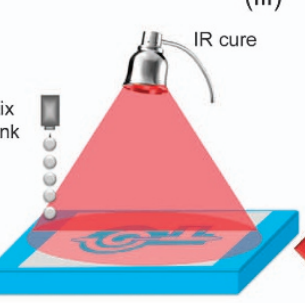

(v)

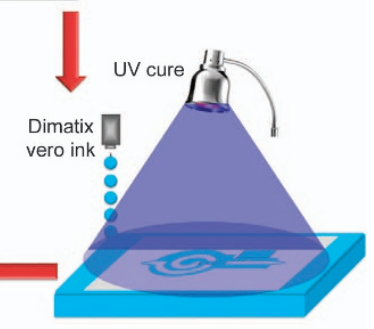

(iv)

Figure 3 Schematic presentation of the fabrication process for multilayer inkjet-printed radio frequency electronics.

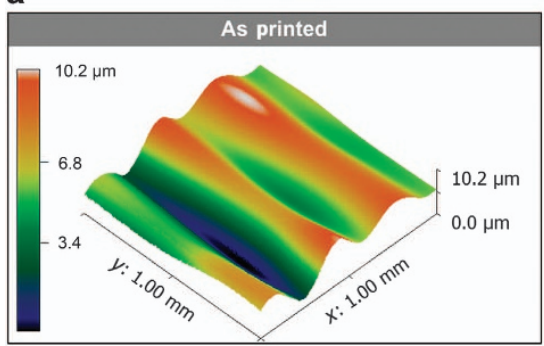

b

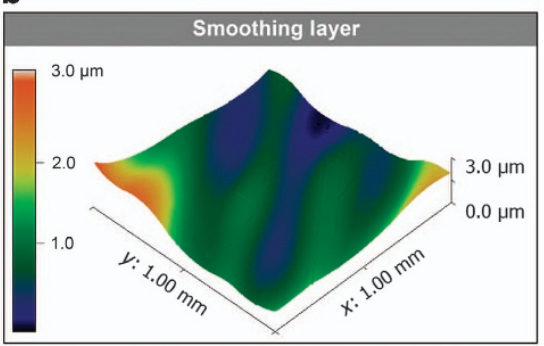

Figure 4 (a) White light interferometer of the 3D printed part showing $1.8 \mu \mathrm{m}$ of RMS roughness. (b) After a smoothing layer of acrylic dielectric ink is applied, there is now $0.4 \mu \mathrm{m}$ of RMS roughness.

connected with crossovers. A cross-section depiction of the process is shown in Figure 3(vi). Note that the silver and dielectric printing (iv-v) could be repeated to create several layers for more complex electronics designs. An image of a fully printed part is shown in Figure 3(vii).

One difficulty to overcome during the fabrication was the wetting of the dielectric VeroWhite ink on the silver layer. The dielectric ink spreads excessively on the solid silver layer underneath. To optimize the wetting condition, a perfluorodecanethiol (PFDT) treatment was used. This treatment has been demonstrated by Tseng et al. to increase the surface hydrophobicity of printed electrodes ${ }^{27}$. The parts are dipped in a bath of $0.28 \mathrm{~mL}$ PFDT with $100 \mathrm{~mL}$ of IPA, then rinsed in IPA and dried with nitrogen. The change can be seen in the contact angle measurement of the dielectric, as is shown in Supplementary Information Figure S3. Without treatment, the ink spreads uncontrollably on the surface (note that all images are taken $5 \mathrm{~s}$ upon impact). After $10 \mathrm{~min}$ of surface treatment, the contact angle was stable at $65^{\circ} \mathrm{C}$, and high definition patterns were possible with the dielectric ink on top of the silver. This allows for the dielectric and silver to be printed in a multilayer fashion capable of fabricating RF passives.

\section{EXPERIMENTAL}

\section{Silver-organo-complex ink formulation}

In an illustrative experiment, a $2 \mathrm{M}$ ethylamine $\left(\mathrm{NH}_{2} \mathrm{CH}_{2} \mathrm{CH}_{3}\right.$, ACS reagent) solution in methanol, which was called 'Complexing Solution \#1', was put in a vial. $10 \mathrm{~mL}$ of ethanolamine
$\left(\mathrm{NH}_{2} \mathrm{CH}_{2} \mathrm{CH}_{2} \mathrm{OH}\right.$, ACS reagent, $\left.\geq 99.0 \%\right)$ and $10 \mathrm{~mL}$ of deionized (DI) water (1:1 ratio) were mixed in another vial. Formic acid $(\mathrm{HCOOH}$, reagent grade, $\geq 95.0 \%)$ was then added to the solution in a drop-wise manner to adjust the solution to a $\mathrm{pH}$ of 10.5. The resulting solution was called 'Complexing Solution \#2'. In another vial, $1 \mathrm{~g}$ of silver acetate $\left(\mathrm{CH}_{3} \mathrm{COOAg}\right.$, ReagentPlus, $\left.99 \%\right)$ was vortex mixed with $2 \mathrm{~mL}$ of complexing solution $\# 1,1.5 \mathrm{~mL}$ of complexing solution \#2, and $0.5 \mathrm{~mL}$ of $2 \%$ 2-hydroxyethylcellulose (2\% 2-HEC in water: methanol $\mathrm{MW}=90000)$ at room-temperature for $30 \mathrm{~s}$, resulting in a light black-colored solution. 2-HEC not only acts as a viscosifier, it also acts as an additive for adhesion of the ink to the substrate. The as-obtained solution was then kept for twelve hours to allow any particles to settle out, yielding a clear supernatant, which was decanted and filtered through a 200-nm syringe filter. This clear and transparent solution, containing approximately $\sim 17 \mathrm{wt} \%$ silver, served as the silver-ethylamineethanolamine-formate complex-based SOC ink $^{25}$.

\section{Characterization of the capacitor, inductor and filter}

Capacitors were measured with an Agilent 4980A LCR meter. The leakage current was measured with a Keithley 4200-SCS. High-frequency measurements of the passive devices were taken in a two-port configuration using 500- $\mu$ m-pitch Z-probes and a cascade probe station with an Agilent E8361A network analyzer. The structural properties were examined using scanning electron microscopy (FEl NovaNano FEG-SEM 630). The thickness and uniformity of printed features on substrates were measured using a surface profiler (Veeco Dektak 150) and 3D interferometry (Zygo, 

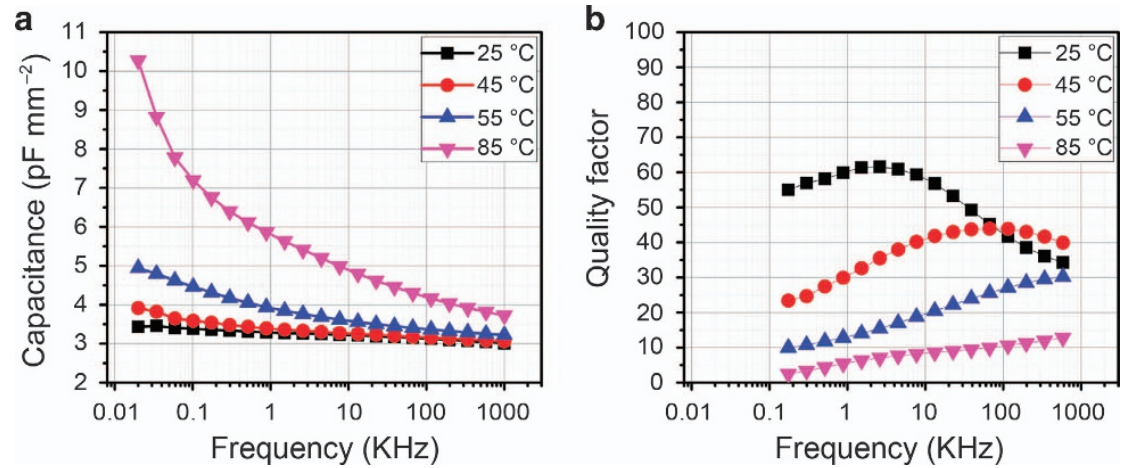

Figure 5 (a) Capacitance at low frequency as a function of temperature, (b) quality factor, tested at $1 \mathrm{~V}$ AC signal and $0 \mathrm{~V}$ bias condition. 11$\mu \mathrm{m}$-thick printed layers.

Newview 7300). The surface tension and viscosity of the inks were measured using a KRUSS DSA100 and Brookfield Rheometer (DV3T). Thermogravimetric analysis was performed using a TG 209 F1 analyzer (Netzsch), with a heating rate of $10^{\circ} \mathrm{C} \mathrm{min}^{-1}$ in air flow.

\section{RESULTS}

\section{Low-frequency capacitor characterization}

To evaluate the process, Metal Insulator Metal capacitors were first printed with the SOC ink for electrodes and the dielectric ink using the process previously described. Capacitors allow for characterization of the leakage current, dielectric, behavior and quality factor at low frequency. The printed capacitors have excellent leakage current values of approximately $1 \times 10^{-10} \mathrm{~A} \mathrm{~cm}^{-2}$ at $0.08 \mathrm{MV} \mathrm{cm}^{-1}$, equivalent to $100 \mathrm{~V}$ across the $11-\mu \mathrm{m}$-thick capacitor (plotted in Supplementary Information Figure S4). The capacitors were also tested against frequency and temperature; see Figures $5 \mathrm{a}$ and $\mathrm{b}$. While the dielectric constant in Figure $5 \mathrm{a}$ is relatively flat with a frequency $(\sim 3)$ up to $45^{\circ} \mathrm{C}$, the material shows considerable low-frequency dispersion at elevated measurement temperatures. This temperature issue can also be seen in the decrease in the quality factor shown in Figure 5b. After cooling to room temperature, the dielectric properties return to the $25^{\circ} \mathrm{C}$ case.

This is a known behavior in acrylic materials, caused from dielectric relaxation at the glass transition temperature, $\left(\sim 50^{\circ} \mathrm{C}\right)$. A thorough investigation of the dielectric relaxation in thin acrylic sheets has been studied by Wubbenhost et al., who described this phenomenon $^{28}$. A complete characterization of the dielectric relaxation with temperature for this material is out of the scope of this work. The important point is that the dielectric properties are sensitive to temperature and should be operated below the glass transition. Humidity impacts the capacitors, providing a 10\% normalized capacitance increase accompanied by a quality factor reduction when tested from $25 \%$ relative humidity to $85 \%$ relative humidity (Supplementary Information Figure S5). The dielectric has also been tested against voltage bias and shows ideal behavior with negligible change in capacitance value or quality factor (Supplementary Information Figure S6). The major changes in both physical and electrical dielectric properties occur at elevated temperature. However, there is no issue as long as the material is fabricated below $80^{\circ} \mathrm{C}$ to avoid excessive warping and operated below $45^{\circ} \mathrm{C}$ to avoid dielectric changes at the glass transition temperature.

\section{High frequency characterization of the capacitor, inductor and filter}

The capacitor, inductor and filter were all measured at high frequencies with a vector network analyzer in a two-port configuration. Electromagnetic models of the devices were created using Ansoft high frequency structure simulator (HFSS) software with the appropriate conductivity, thickness and dielectric properties to compare with measurements. From the network analyzer, the measured S-parameters are de-embedded with an open-short method and are converted to $Y$-parameters at each frequency point $f$, which is a standard procedure ${ }^{29,30}$. The following equations are used to convert to capacitance $C$, inductance $L$, and quality factor $Q$.

$$
\begin{gathered}
C=\frac{1}{\operatorname{im}\left(\frac{4}{Y_{11}+Y_{22}-Y_{12}-Y_{21}}\right) 2 \pi f} \\
L=\frac{\operatorname{im}\left(\frac{4}{Y_{11}+Y_{22}-Y_{12}-Y_{21}}\right)}{2 \pi f} \\
Q=-\left(\frac{\operatorname{im}\left(Y_{11}+Y_{22}-Y_{12}-Y_{21}\right)}{\operatorname{re}\left(Y_{11}+Y_{22}-Y_{12}-Y_{21}\right)}\right)
\end{gathered}
$$

Figures $6 a$ and $b$ show the quality factor and the capacitance value as a function of frequency. The capacitor is $2 \mathrm{pF}$ and has a self-resonant frequency of $6.5 \mathrm{GHz}$. The quality factor of the capacitor starts out at 25 and drops down to zero at selfresonance as expected. The quality factor results are also consistent with the measured devices at lower frequency and $25^{\circ} \mathrm{C}$, as shown in Figure $5 \mathrm{~b}$. It should be noted that previous printed dielectrics have had difficulty achieving such high quality factors in the high $\mathrm{MHz}$ and $\mathrm{GHz}$ regime, typically reporting Q's lower than 10, largely due to the loss tangent of the dielectrics used $^{8,9}$.

An inductor has also been tested, as shown in Figures $6 \mathrm{c}$ and $\mathrm{d}$. This 1.5 turn inductor has an outer diameter of $4 \mathrm{~mm}$ with a $600-\mu \mathrm{m}$-thick spiral, has a self-resonance at $4 \mathrm{GHz}$ and is approximately $8 \mathrm{nH}$ at a frequency of $1 \mathrm{GHz}$. The quality factor in simulation is slightly higher than the measured results in Figure $6 \mathrm{~d}$. This is likely due to there being greater resistance from the printed silver ink than simulated; the inductors are extremely sensitive to the conductivity and thickness of the printed ink. These inductors have been printed with five layers of SOC ink and show a peak quality factor of $\sim 8$. The quality factors for both the inductors and capacitors are considered state-of-the-art among inkjet-printed passives, even with the low $80^{\circ} \mathrm{C}$ processing temperature ${ }^{8,9}$.

The capacitor and inductor were implemented in a classic Butterworth low pass filter with a cutoff frequency of 2.0 GHz (Ref. 31). The Butterworth filter provides a maximally flat passband, and the low pass filter was designed in a full-wave EM simulation in Ansoft HFSS to finalize the layout. Figure 7a shows a microscopy image of the fabricated filter. There is an inset 

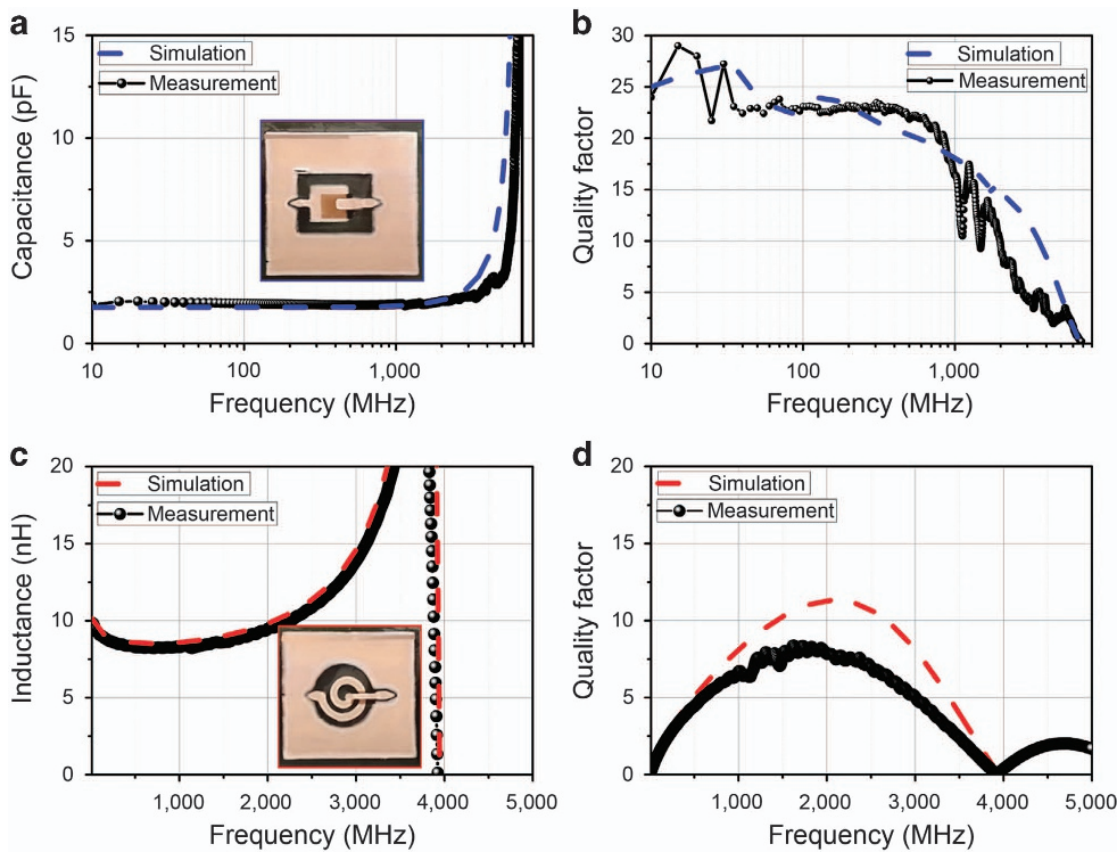

Figure 6 (a) Capacitor value measured and simulated, (b) quality factor (capacitor area is $\sim 0.9 \mathrm{~mm}^{2}$ with an $11-\mu \mathrm{m}$ dielectric), (c) inductor value measured and simulated, and (d) quality factor (1.5 turn inductor with an outer radius of $4 \mathrm{~mm}$ and $600-\mu \mathrm{m}$-thick lines).

a
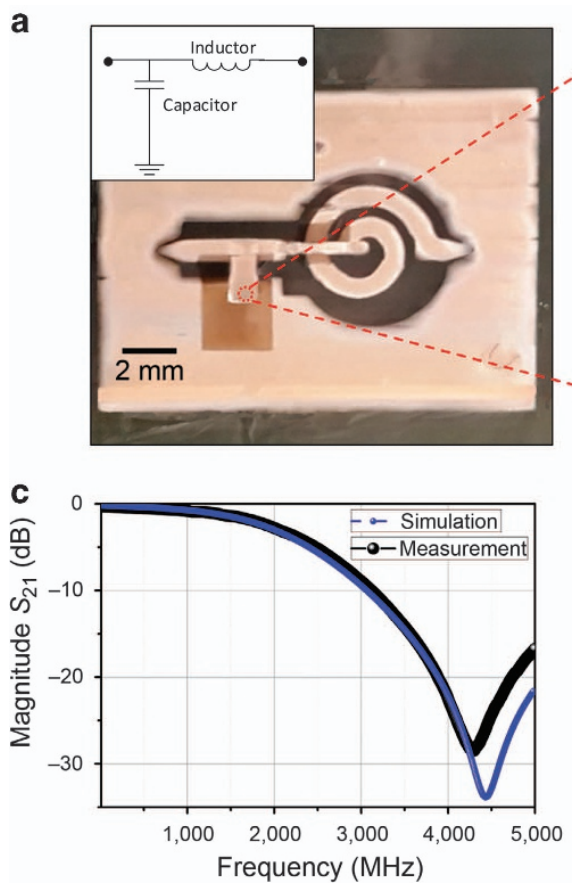
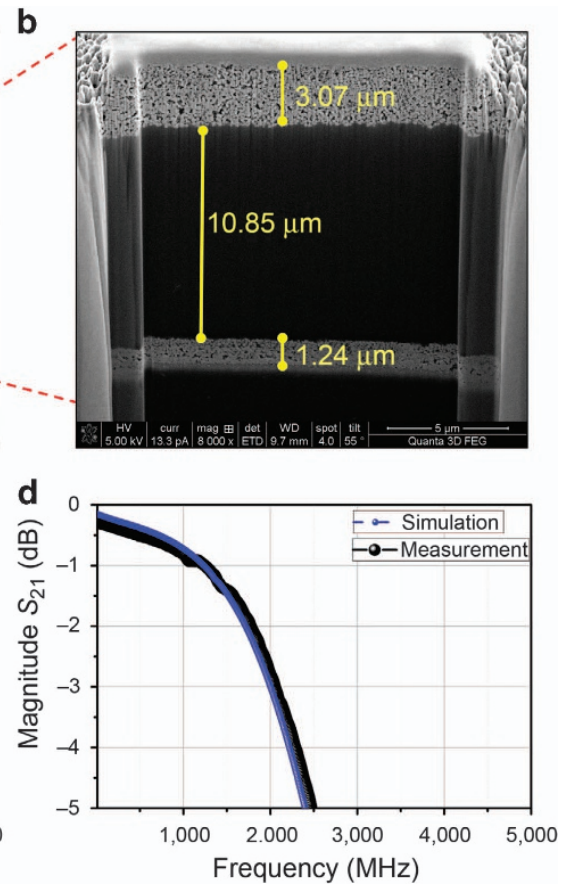

Figure 7 (a) Microscope image of the printed filter. (b) SEM focused ion beam cross-section image through the capacitor area, showing the thickness of the printed dielectric and the top and bottom electrodes of the filter. (c) Measured and simulated $S_{21}$ filter response versus frequency. (d) Zoomed in view of the filter response.

depicting the corresponding placement of the capacitor and inductor for clarity. The capacitor area is further visualized by the cross-sectional SEM image in Figure 7b. From the cut, the printed silver thickness at both layers and the 11- $\mu$ m-thick dielectric spacing of the capacitor are clear. The measured frequency response of the filter in Figures 7c and $d$ matches well with the HFSS simulation with a $3-\mathrm{dB}$ cutoff at $2 \mathrm{GHz}$. There is signal rejection near $10 \mathrm{~dB}$ at $3 \mathrm{GHz}$ and better than $20 \mathrm{~dB}$ at $4 \mathrm{GHz}$. The filter has no ripple in the passband, as expected, and an insertion loss of $0.8 \mathrm{~dB}$ at $1 \mathrm{GHz}$ from the zoomed-in response of Figure $7 \mathrm{~d}$. The low insertion loss is excellent for a first ever fully inkjet-printed lumped element filter, especially considering the temperature constraints of $80^{\circ} \mathrm{C}$. The performance is state of the art, even compared with previous 2D distributed printed filters where nanoparticles are printed on a standard substrate, reporting insertion losses ranging from 3.6 to $10 \mathrm{~dB}$ (Refs. 11-15). 


\section{DISCUSSION}

Inkjet printing is transitioning from solely a graphic arts medium into a useful fabrication tool. The ability to deposit multiple materials and the scalability of inkjet systems with hundreds of nozzles makes it possible to realize large and complex parts. A process is presented in this work in which inkjet-printed UV-cured polymer and silver ink are integrated together. A major challenge is integrating a metal with the low-temperature UV-cured plastic material. A novel SOC ink has been deployed, developed in-house, which provides a conductivity of $1 \times 10^{7} \mathrm{~S} \mathrm{~m}^{-1}$ at $80^{\circ} \mathrm{C}$ to combine the materials effectively. The combination of quick (5-minute) IR curing of the silver and rapid UV curing of the polymer in an ambient environment makes this an attractive method for fabrication. The capacitor and inductor exhibit state-of-the-art quality factors of $\sim 20$ and 8 , respectively, in the radio frequency regime and compare well with electromagnetic simulation models. By implementing these components, a low pass filter has been fabricated with an insertion loss of $0.8 \mathrm{~dB}$ at $1 \mathrm{GHz}$. This is excellent considering that this is the first demonstration of a fully inkjet-printed lumped element filter. Although there is still room for improvement in terms of the conductivity of the metal, loss tangent of the dielectric, and temperature range of the printed acrylic, this is a significant step forward.

\section{ACKNOWLEDGEMENTS}

Garret McKerricher and Dr Mohammad Vaseem contributed equally to this work. We acknowledge financial support from King Abdullah University of Science and Technology (KAUST) Office of Sponsored Research (OSR). For assistance and training with the Objet printer, we greatly appreciate the support of Rahman M. Hasan at the Visualization Facilities at KAUST. We would also like to thank Nini Wei, Long Chen and Shuai Yang for their work and assistance with SEM analysis of the films.

\section{COMPETING INTERESTS}

The authors declare no conflict of interest.

\section{REFERENCES}

1 Snyder T, Andrews $M$, Weislogel $M$ et al. 3D systems' technology overview and new applications in manufacturing. 3D Printing and Additive Manufacturing 2014; 1: 169-176.

2 Wu S-Y, Yang C, Hsu W et al. 3D-printed microelectronics for integrated circuitry and passive wireless sensors. Microsystems and Nanoengineering 2015; 1: 15013.

3 Chieh J-CS, Dick B, Loui S et al. Development of a Ku-band corrugated conical horn using 3-D print technology. IEEE Antennas and Wireless Propagation Letters 2014; 13: 201-204.

4 Nayeri $\mathrm{P}$, Liang $\mathrm{M}$, Sabory-Garcia RA et al. 3D printed dielectric reflectarrays: Low-cost high-gain antennas at sub-millimeter waves. IEEE Antennas and Wireless Propagation Letters 2014; 62: 2000-2008.

5 Tsang $\mathrm{HH}$, Barton JH, Rumpf RC et al. Effects of extreme surface roughness on 3D printed horn antenna. Electronics Letters 2013; 49: 734-736.

6 Mckerricher G, Titterington D, Shamim A. A fully inkjet printed 3D honeycomb inspired patch antenna. IEEE Antennas and Wireless Propagation Letters 2015; 15 : 1.

7 Redinger D, Molesa S, Yin S et al. An ink-jet-deposited passive component process for RFID. IEEE Transactions on Electron Devices 2004; 51: 1978-1983.

8 McKerricher G, Perez JG, Shamim A. Fully inkjet printed RF inductors and capacitors using polymer dielectric and silver conductive ink with through vias. IEEE Transactions on Electron Devices 2015; 62: 1002-1009.

9 Cook BS, Mariotti C, Cooper JR et al. Inkjet-printed, vertically-integrated, highperformance inductors and transformers on flexible LCP substrate. 2014 IEEE MTT-S International Microwave Symposium (IMS 2014); 1-6 Jun 2014; Tampa, FL, USA; 2014: 1-4.

10 Menicanin AB, Zivanov LD, Damnjanovic MS et al. Low-cost CPW meander inductors utilizing ink-jet printing on flexible substrate for high-frequency applications. IEEE Transactions on Electron Devices 2013; 60: 827-832.
11 Sette D, Blayo A, Poulain C et al. Silver nanoparticle inkjet printed 17 GHz filter. 2013 IEEE MTT-S International Microwave Symposium Digest (IMS 2013); 2013: 1-4.

12 Sriprachuabwong C, Srichan C, Lomas T et al. Simple RC low pass filter circuit fabricated by unmodified desktop inkjet printer. International Conference on Electrical Engineering/Electronics Computer Telecommunications and Information Technology (ECTI-CON 2010); 2010: 929-932.

13 Ahmad W, Budimir D, Maric A et al. Inkjet printed bandpass filters and filtennas using silver nanoparticle ink on flexible substrate. IEEE International Symposium on Antennas and Propagation USNC/URSI National Radio Science Meeting 2015; 19-24 Jul 2015; Vancouver, BC, Canada; 2015: 145-146.

14 Arabi E, McKerricher G, Shamim A. Comparison of filters: Inkjet printed on PEN substrate versus a laser-etched on LCP substrate. 2014 44th European Microwave Conference (EuMC 2014); 5 Oct 2015; Rome, Italy; 2014: 1226-1229.

$15 \mathrm{Kao} \mathrm{HL}$, Cho CL, Chang LC. Inkjet-printed interdigital coupled line filter on liquid crystal polymer substrate. IEEE Electron Device Letters 2013; 34: 1584-1586.

16 Sridhar A, Reiding J, Adelaar $\mathrm{H}$ et al. Inkjet-printing- and electroless-plating- based fabrication of RF circuit structures on high-frequency substrates. Journal of Micromechanics Microengineering 2009; 19: 85020.

17 Magdassi Seditor. The Chemistry of Inkjet Inks. World Scientific: Singapore; Hackensack, NJ, USA. 2010, 345.

18 Stratasys. Material saftey data sheet-objet VeroWhite plus. Report No.: RGD835; cited Nov 9 2015; Stratasys; 2014. www.Stratasys.com.

19 Stratasys. Polyjet material specifications-VeroWhite plus. Cited Nov 9 2015; 2014. www.stratasysdirect.com.

20 Djordjevic AR, Likar-Smiljanic VD, Sarkar TK. Wideband frequency-domain characterization of FR-4 and time-domain causality. IEEE Transactions on Electromagnetic Compatibility 2001; 43: 662-667.

21 Walker SB, Lewis JA. Reactive silver inks for patterning high-conductivity features at mild temperatures. Journal of the American Chemical Society 2012; 134: 1419-1421.

22 Dong Y, Li X, Liu S et al. Facile synthesis of high silver content MOD ink by using silver oxalate precursor for inkjet printing applications. Thin Solid Films 2015; 589: 381-387.

23 Hong CM, Wagner S. Inkjet printed copper source/drain metallization for amorphous silicon thin-film transistors. IEEE Electron Device Letters 2000; 21: 384-386.

24 Walker SB, Ahn BY, Lewis JA. Reactive Silver Inks for High-performance Printed Electronics. Sigma Aldrich Technical Documents); Sigma Aldrich; 2015.

25 Vaseem M, McKerricher G, Shamim A. Robust design of a particle-free silver-organo-complex ink with high conductivity and inkjet stability for flexible electronics. ACS Applied Materials and Interfaces 2016; 8: 177-186.

26 Derby B. Inkjet printing of functional and structural materials: Fluid property requirements, feature stability, and resolution. Annual Review of Materials Research 2010; 40: 395-414.

27 Tseng H-Y. Scaling of Inkjet-printed transistors using novel printing techniques. EECS Department, University of California, Berkeley; 2011. http://www.eecs.berkeley.edu/Pubs/TechRpts/2011/EECS-2011-146.html.

28 Wubbenhorst M, Murray CA, Forrest JA et al. Dielectric relaxations in ultra-thin films of PMMA: Assessing the length scale of cooperativity in the dynamic glass transition. ISE Proceedings 11th International Symposium on Electrets; 3 Oct 2002; Melbourne, Victoria, Australia; 2002: 401-406.

29 Frickey DA. Conversions between S, Z, Y, H, ABCD, and T parameters which are valid for complex source and load impedances. IEEE Transactions on Microwave Theory and Techniques 1994; 42: 205-211.

30 Okada K, Masu K. Modeling of spiral inductors. In: Zhurbenko V, editor. Advanced Microwave Circuits and Systems. Cited Dec 24 2014; InTech; 2010. http://www. intechopen.com/books/advanced-microwave-circuits-and-systems/modeling-ofspiral-inductors.

31 Butterworth S. On the theory of filter amplifiers. Experimental Wireless and the Wireless Engineer 1930; 7: 536-541.

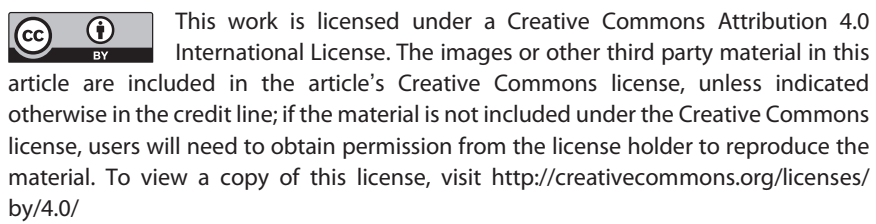

(c) The Author(s) 2017

Supplementary Information for this article can be found on the Microsystems \& Nanoengineering website (http://www.nature.com/ micronano) 